\title{
A nomogram for predicting bowel obstruction in preoperative colorectal cancer patients with clinical characteristics
}

\author{
Xinger $\mathrm{LV}^{\dagger}$, Hong Yu${ }^{\dagger}$, Peng Gao, Yongxi Song, Jingxu Sun, Xiaowan Chen, Yu Wang and Zhenning Wang ${ }^{*}$ (D)
}

\begin{abstract}
Background: Bowel obstruction (BO) is a complication that commonly affects patients with colorectal cancer (CRC). $\mathrm{BO}$ causes severe outcomes, and its treatment leads to a dilemma for many surgeons. Moreover, the factors correlated to $\mathrm{BO}$ in preoperative CRC patients remain unclear. The objectives of this study were to investigate the clinical characteristics of $\mathrm{BO}$ to identify risk predictors and to construct a $\mathrm{BO}$ prediction model with preoperative CRC patients.

Methods: A large-scale, retrospective cohort, population-based study analyzed the data of 11,814 patients obtained from the Surveillance, Epidemiology, and End Results and Medicare claims-linked databases (SEER-M database). Patients aged $\geq 66$ years and primarily diagnosed with CRC from 1992 to 2009 were divided into BO and non-BO groups. Cox proportional hazards regression models were used to determine predictors, and then, a nomogram was constructed by those predictors.

Results: A total of 11,814 patients (5293 men and 6251 women) were identified. In multivariate analysis, 14 factors were found to be associated with BO including age, race, marital status, residence location, T category, M category, primary tumor site, histologic type, histologic grade, tumor size, history of alcoholism, chemotherapy, radiotherapy, abdominal pain, and anemia. A nomogram predicting the 90- and 180-day rates of BO was built for the preoperative CRC patients with a C-index of 0.795 .

Conclusions: This study identified $14 \mathrm{BO}$-related factors, and a statistical model was constructed to predict the onset of $\mathrm{BO}$ in preoperative CRC patients. The obtained data may guide decision-making for the intervention of patients at risk for $\mathrm{BO}$.
\end{abstract}

Keywords: Colorectal cancer, Bowel obstruction, Nomograms, Risk factors, SEER program

\section{Background}

Colorectal cancer (CRC) is the third most common cancer in both men and women in the USA [1]. Despite the high percentage of patients undergoing screening colonoscopy at the appropriate age in the USA, a large number of patients present with advanced-stage CRC [2, 3], some of whom require chemotherapy or radiotherapy before tumor resection or require palliative treatment. Before surgery, it is possible to have a complication that can lead to severe results. One such complication is

\footnotetext{
* Correspondence: josieon826@sina.cn

${ }^{+}$Xinger Lv and Hong Yu contributed equally to this work.

Department of Surgical Oncology and General Surgery, The First Affiliated

Hospital of China Medical University, 155 North Nanjing Street, Heping

District, Shenyang City 110001, People's Republic of China
}

bowel obstruction (BO), and $25-40 \%$ of CRC patients suffer from this condition $[4,5]$.

$\mathrm{BO}$ symptoms at onset are insidious and subtle and can be easily ignored in clinical practice. In this way, once patients get $\mathrm{BO}$, they often present with intractable nausea, vomiting, and dehydration [6-8], which cause considerable distress to patients and their families $[9,10]$. Some studies have reported that elective surgery for $\mathrm{BO}$ offered better results $[11,12]$. However, other studies indicate that $\mathrm{BO}$ has a poor prognosis even with interventions [4, 13-15]. These conflicting results often put both physicians and surgeons in an ethical dilemma. Therefore, it is critical to predict the onset of $\mathrm{BO}$ and identify specific

(c) The Author(s). 2019 Open Access This article is distributed under the terms of the Creative Commons Attribution 4.0 International License (http://creativecommons.org/licenses/by/4.0/), which permits unrestricted use, distribution, and 
populations that need to be monitored carefully or can benefit from prophylactic treatments.

The objectives of this study were to conduct a population-based study to evaluate factors associated with $\mathrm{BO}$ and to build a statistical model to predict the development of BO by using data from the Surveillance, Epidemiology, and End Results and Medicare claimslinked databases (SEER-M database). Our findings may have particular value for patients with potential risk of $\mathrm{BO}$ and may assist clinicians in appropriate decision-making in surgical intervention.

\section{Materials and methods \\ Data source}

This retrospective study used data from the National Cancer Institute Surveillance, Epidemiology, and End Results (SEER) registry linked with Medicare claims data [16]. The SEER database is a population-based cancer registry covering approximately $28 \%$ of the population across the USA [17]. The Centers for Medicare and Medicaid administers Medicare, which is the primary health insurance program for approximately $97 \%$ of the population of the USA aged $\geq 66$ years [16].

\section{Eligibility criteria}

The inclusion criteria for eligible patients were as follows:

1. Age $\geq 66$ years and primary diagnosis of CRC (SEER cancer site codes 18.0, 18.2-18.9, 19.9, and 20.9) from 1992 to 2009.

2. Having a record for BO (ICD-9 code 560.89 and 560.9 , absence of intestinal or peritoneal adhesions with obstruction) [18] after diagnosis of CRC and before the execution of cancer-related operations (if received), as well as no previous history of BO.

3. No record of BO in overall survival time and an absence of cancer-related operations after the diagnosis of CRC.

The exclusion criteria were as follows:

1. Having a diagnosis of $\mathrm{CRC}$ or other cancers within 1 year after the first admission.

2. Having a record of any cancer-related surgery between $\mathrm{CRC}$ diagnosis record and $\mathrm{BO}$ record if in the $\mathrm{BO}$ group.

3. Having a record of any cancer-related surgery after cancer diagnosis if in the non-BO group.

4. Having a diagnosis of ulcerative colitis (ICD-9 codes 556.X) or Crohn's disease (ICD-9 codes 555.X) because these conditions are risk factors for $\mathrm{CRC}$ and may require therapies distinct from those used in populations not affected by these two diseases [19, 20].
5. Lack of full coverage through Medicare parts A and B from 12 months before diagnosis to 60 months after diagnosis (in cases in which the patients survived) or enrollment in a health maintenance organization (HMO).

6. Having a $\mathrm{BO}$ record within 30 days of $\mathrm{CRC}$ diagnosis because we considered that a $\mathrm{BO}$ record was present at diagnosis (to evaluate two medical interventions happening at different times) [20, 21].

\section{Study variables}

Demographic and clinical information were extracted from the SEER patient entitlement and diagnosis summary file at the time of diagnosis. The demographic variables included year of diagnosis, age, gender, race, marital status, and residence location. Socioeconomic status (household income and education level) data were categorized into quadrants. The primary tumor site was classified as the rectum, the left-side colon (including the splenic flexure and the descending and sigmoid colons), and the right-side colon (including the cecum, the ascending colon, the hepatic flexure and the transverse colon). Other tumor characteristics including histologic grade, histologic type (adenocarcinoma, mucinous carcinoma, signet-ring cell carcinoma), tumor size, and $\mathrm{T}$ and $M$ categories were assessed using the eighth edition of the American Joint Committee on Cancer (AJCC) TNM staging system [22]. Concomitant symptoms that developed 1 year before cancer diagnosis, including abdominal pain and changes in bowel habits, among others, were also included in this study (all symptom codes used in this study are listed in Additional file 1: Table S1).

All patients who received chemotherapy between diagnosis of CRC and BO, or within 180 days after diagnosis of CRC (if no BO record), were identified. The adjuvant chemotherapy regimens were 5-FU/capecitabine alone or 5-FU/capecitabine plus oxaliplatin (FOLFOX/CapeOX) or 5-FU/capecitabine plus irinotecan (FOLFIRI/XELIRI). Patients who received bevacizumab were separated from the FOLFOX/CapeOX and FOLFIRI/XELIRI groups and were included in two other groups. Patients who received chemotherapy but were not included in these five groups were assigned to another group. The remaining patients with no chemotherapy records were included in the nonchemotherapy group. In addition, the FOLFOX/CapeOX group included patients with any record of oxaliplatin within 30 days of the first chemotherapy dose [23]. This criterion was applicable to other groups that received more than one drug. The radiotherapy group included patients with radiotherapy records between diagnosis of CRC and BO or within 180 days after a diagnosis of CRC (in cases of absence of $\mathrm{BO}$ records). The remaining patients were included in the nonradiotherapy group 
(all treatment codes used in this study are listed in Additional file 2: Table S2).

\section{Comorbidities}

To take comorbidities into account, we used the Centers for Medicare and Medicaid Service's Hierarchical Condition Category to assess the health conditions of patients [24] and collapsed it into quadrants following common practice. The Medicare claims pertaining to comorbidities found during the 12 months before cancer diagnosis were also considered.

\section{Statistical analysis}

Demographic and clinical variables among $\mathrm{BO}$ and non-BO groups were compared using the $\chi^{2}$ and Mann-Whitney tests. Cox proportional hazards regression models were used in both univariate and multivariate analyses to evaluate relationships between the time-to- $\mathrm{BO}$ (the models set the date of cancer diagnosis as time 0 and treated death and loss to follow-up as censoring events) and factors that contributed to BO. Only significant BO-related variables in univariate analysis were included in multivariate analysis and were adjusted for potential confounders using stepwise backward selection. A curve of cumulative $\mathrm{BO}$ rate was built using Kaplan-Meier survival analysis and log-rank tests. A nomogram was formulated on the basis of all identified independent predictors and was constructed for predicting the 90- and 180-day rates of BO. Calibration was done by comparing nomogram-predicted versus observed outcomes, and accuracy was calculated by the $\mathrm{C}$-index. Afterwards, the model was rectified by a 10 -fold cross-validation to reduce the bias from random sampling of the training set. Nine tenths of the patients were randomly assigned to the training set, and one tenth was assigned to the validation set ten times, and the mean C-index was calculated to assess the model [25].

All statistical analyses and graphs were performed using $\mathrm{R}$ software version 3.3.1 ( $\mathrm{R}$ Foundation for statistical computing, Vienna, Austria), SAS version 9.4 (SAS Institute, Cary, NC, USA), and PASW Statistics version 22.0 (SPSS, Inc., Somers, NY, USA). For all analyses, $p$ values less than 0.05 were considered statistically significant.

\section{Results}

From 1992 to 2009, 11,814 patients with a primary diagnosis of CRC were identified from the SEER-M database to serve as the study population. Among patients who met the inclusion criteria, $3104(26.3 \%)$ patients with a diagnosis of $\mathrm{BO}$ (no previous $\mathrm{BO}$ record before the study period and no cancer-related surgery before the onset of BO) were classified as the BO group. Patients without $\mathrm{BO}$ and not subjected to cancer surgery were classified as the non- $\mathrm{BO}$ group. The median survival time was 270 days, and the median onset of $\mathrm{BO}$ was 55 days.

\section{Overall comparison of the $\mathrm{BO}$ and non-BO groups}

Detailed patient baseline characteristics at the time of diagnosis are shown in Table 1. The population consisted of 5293 men and 6251 women. The rate of BO decreased over the study period: $38.3 \%$ in 1992-1996, $26.3 \%$ in $1997-2001,24.8 \%$ in $2002-2005$, and $24.0 \%$ in 2006-2009 ( $p<0.001$ for the trend).

The effect of the time-to-BO was considered in univariate analysis by using Cox proportional hazards regression models (Table 2). Socioeconomic status, including income and education level, was not significantly different between the two groups $(p=0.107$ and 0.571 , respectively), race and marital status were associated with $\mathrm{BO}$, older patients were more likely to develop $\mathrm{BO}(p<$ 0.001 ; Fig. 1a), men were more likely to present with $\mathrm{BO}$ than women ( $27.6 \%$ and $25.2 \%$, respectively $p=0.02$ ), living in a large urban area also appeared to affect the likelihood of developing BO. However, data on gender and residence location were later removed from multivariate analysis. Tumor characteristics were analyzed in cases in which they contributed to the development of BO. All tumor characteristics, including $\mathrm{T}$ category $(p<0.001$, Fig. $1 b), M$ category $(p<0.001$, Fig. 1 c), primary tumor site $(p<0.001$, Fig. $1 \mathrm{~d})$, histologic type $(p<0.001$, Fig. 2a), histologic grade $(p<0.001$, Fig. $2 \mathrm{~b})$, and tumor size $(p<0.001$, Fig. $2 \mathrm{c})$, associated with BO. Cancer-related symptoms that occurred 1 year before CRC diagnosis were also included in the analysis. Seven symptoms including abdominal pain, abdominal mass, anemia, nutritional deficiency, change of bowel habits, hemorrhage, and loss of appetite were associated with BO.

All the predictors confirmed in multivariate analysis are listed in Table 2. Multivariate Cox proportional hazards models produced results similar to those of univariate analysis: the rate of $\mathrm{BO}$ was decreased as age was increased, and the adjusted hazard ratio (HR) for $\mathrm{BO}$ among the age group 66-70 years was 1.737 (HR [95\% CI, 1.558-1.935]) compared with 1.765 (HR [95\% CI, 1.596-1.953]) in the age group 71-75 years, $1.523 \mathrm{HR}$, (95\% CI, 1.384-1.677) in the age group 76-80 years, and 1.000 in the age group $\geq 81$ years $(p<0.001$ for trend). The patients who developed $\mathrm{BO}$ tended to be Asian (HR, 1.062 [95\% CI, 0.887-1.271]) and married (HR, 1.115 [95\% CI, 1.028-1.208]). All evaluated tumor characteristics played an important role in BO. After data adjustment, patients with tumors in the T4a category (HR, 9.064 [95\% CI, 6.824-12.039]), unknown M category (HR, 1.213 [95\% CI, 1.108-1.328]), and left-side colon (HR, 2.093 [95\% CI, 1.892-2.315]) and with poorly differentiated histologic grade (HR, 1.131 [95\% CI, 1.022-1.251]), mucinous carcinoma (HR, 1.593 [95\% CI, 1.392-1.823]), 
Table 1 Characteristics of patients with CRC stratified by BO

\begin{tabular}{|c|c|c|c|}
\hline Patient characteristics & Overall (N\%) & No BO (N\%) & BO (N\%) \\
\hline \multicolumn{4}{|l|}{ Gender } \\
\hline Male & $5293(44.8 \%)$ & 3831 (44.0\%) & $1462(47.1 \%)$ \\
\hline Female & $6521(55.2 \%)$ & 4879 (56.0\%) & $1642(52.9 \%)$ \\
\hline \multicolumn{4}{|l|}{ Age at diagnosis, years } \\
\hline $66-70$ & 1525 (12.9\%) & $966(11.1 \%)$ & 559 (18.0\%) \\
\hline $71-75$ & 1975 (16.7\%) & 1296 (14.9\%) & $679(21.9 \%)$ \\
\hline $76-80$ & $2342(19.8 \%)$ & $1617(18.6 \%)$ & $725(23.4 \%)$ \\
\hline$>80$ & $5972(50.6 \%)$ & $4831(55.5 \%)$ & $1141(36.8 \%)$ \\
\hline \multicolumn{4}{|l|}{ Race } \\
\hline White & $9421(79.7 \%)$ & $6945(79.7 \%)$ & $2476(79.8 \%)$ \\
\hline Black & 1566 (13.3\%) & $1185(13.6 \%)$ & $381(12.3 \%)$ \\
\hline Asian & 372 (3.1\%) & $244(2.8 \%)$ & $128(4.1 \%)$ \\
\hline Other & 455 (3.9\%) & $336(3.9 \%)$ & 119 (3.8\%) \\
\hline \multicolumn{4}{|l|}{ Marital status } \\
\hline Single + separated & 1358 (11.5\%) & $1023(11.7 \%)$ & $335(10.8 \%)$ \\
\hline Married & $4441(37.6 \%)$ & 3015 (34.6\%) & $1426(45.9 \%)$ \\
\hline Divorced + widowed & $5296(44.8 \%)$ & $4073(46.8 \%)$ & $1223(39.4 \%)$ \\
\hline Other & 719 (6.1\%) & 599 (6.9\%) & $120(3.9 \%)$ \\
\hline \multicolumn{4}{|l|}{ Residence location* } \\
\hline Big metro & $6750(57.2 \%)$ & 4869 (55.9\%) & $1881(60.6 \%)$ \\
\hline Metro or urban & 3814 (32.3\%) & 2886 (33.1\%) & $928(29.9 \%)$ \\
\hline Less urban or rural & $1247(10.6 \%)$ & $952(10.9 \%)$ & 295 (9.5\%) \\
\hline \multicolumn{4}{|l|}{ Median household income } \\
\hline 1st quartile & $2781(23.5 \%)$ & $2094(24.0 \%)$ & $687(22.1 \%)$ \\
\hline 2nd quartile & $2791(23.6 \%)$ & 2058 (23.6\%) & $733(23.6 \%)$ \\
\hline 3rd quartile & $2786(23.6 \%)$ & 2037 (23.4\%) & $749(24.1 \%)$ \\
\hline 4th quartile & $2791(23.6 \%)$ & 2015 (23.1\%) & $776(25.0 \%)$ \\
\hline Unknown & $665(5.6 \%)$ & $506(5.8 \%)$ & 159 (5.1\%) \\
\hline \multicolumn{4}{|l|}{ Level of education } \\
\hline 1st quartile & $2770(23.4 \%)$ & 2032 (23.3\%) & $738(23.8 \%)$ \\
\hline 2nd quartile & $2817(23.8 \%)$ & 2068 (23.7\%) & $749(24.1 \%)$ \\
\hline 3rd quartile & 2798 (23.7\%) & $2061(23.7 \%)$ & 737 (23.7\%) \\
\hline 4th quartile & $2762(23.4 \%)$ & $2043(23.5 \%)$ & $719(23.2 \%)$ \\
\hline Unknown & $667(5.6 \%)$ & $506(5.8 \%)$ & $161(5.2 \%)$ \\
\hline \multicolumn{4}{|l|}{ Year of diagnosis } \\
\hline 1992-1996 & $1228(10.4 \%)$ & $758(8.7 \%)$ & $470(15.1 \%)$ \\
\hline 1997-2001 & 1999 (16.9\%) & $1478(17.0 \%)$ & $521(16.8 \%)$ \\
\hline $2002-2005$ & $3752(31.8 \%)$ & 2796 (32.1\%) & $956(30.8 \%)$ \\
\hline $2006-2009$ & 4835 (40.9\%) & 3678 (42.2\%) & $1157(37.3 \%)$ \\
\hline \multicolumn{4}{|l|}{ Tumor characteristics } \\
\hline \multicolumn{4}{|l|}{ T category } \\
\hline Tis & 595 (5.0\%) & 491 (5.6\%) & $104(3.4 \%)$ \\
\hline $\mathrm{T} 1$ & 2054 (17.4\%) & 1662 (19.1\%) & $392(12.6 \%)$ \\
\hline $\mathrm{T} 2$ & 307 (2.6\%) & $126(1.4 \%)$ & $181(5.8 \%)$ \\
\hline
\end{tabular}


Table 1 Characteristics of patients with CRC stratified by BO (Continued)

\begin{tabular}{|c|c|c|c|}
\hline Patient characteristics & Overall (N\%) & No BO (N\%) & $\mathrm{BO}(\mathrm{N} \%)$ \\
\hline T3 & $1647(13.9 \%)$ & $596(6.8 \%)$ & $1051(33.9 \%)$ \\
\hline T4a & $161(1.4 \%)$ & $35(0.4 \%)$ & $126(4.1 \%)$ \\
\hline $\mathrm{T} 4 \mathrm{~b}$ & 717 (6.1\%) & $457(5.2 \%)$ & $260(8.4 \%)$ \\
\hline Unknown & $6333(53.6 \%)$ & $5343(61.3 \%)$ & 990 (31.9\%) \\
\hline \multicolumn{4}{|l|}{ M category } \\
\hline Mo & $2475(20.9 \%)$ & 1686 (19.4\%) & $789(25.4 \%)$ \\
\hline M1 & $3311(28.0 \%)$ & $2684(30.8 \%)$ & $627(20.2 \%)$ \\
\hline Unknown & $6028(51.0 \%)$ & $4340(49.8 \%)$ & $1688(54.4 \%)$ \\
\hline \multicolumn{4}{|l|}{ Primary tumor site } \\
\hline Rectum & $4674(39.6 \%)$ & 3666 (42.1\%) & $1008(32.5 \%)$ \\
\hline Left-sided colon & $2624(22.2 \%)$ & $1648(18.9 \%)$ & $976(31.4 \%)$ \\
\hline Right-sided colon & $4516(38.2 \%)$ & 3396 (39.0\%) & $1120(36.1 \%)$ \\
\hline \multicolumn{4}{|l|}{ Histologic type } \\
\hline Adenocarcinoma & $11,200(94.8 \%)$ & 8375 (96.2\%) & $2825(91.0 \%)$ \\
\hline Mucinous carcinoma & $523(4.4 \%)$ & $281(3.2 \%)$ & $242(7.8 \%)$ \\
\hline Signet-ring cell carcinoma & $91(0.8 \%)$ & $54(0.6 \%)$ & $37(1.2 \%)$ \\
\hline \multicolumn{4}{|l|}{ Histologic grade } \\
\hline Well & $724(6.1 \%)$ & $509(5.8 \%)$ & $215(6.9 \%)$ \\
\hline Moderate & $5011(42.4 \%)$ & 3327 (38.2\%) & $1684(54.3 \%)$ \\
\hline Poor & $1488(12.6 \%)$ & $973(11.2 \%)$ & $515(16.6 \%)$ \\
\hline Undifferentiated & $96(0.8 \%)$ & $69(0.8 \%)$ & $27(0.9 \%)$ \\
\hline Unknown & 4495 (38.0\%) & $3832(44.0 \%)$ & $663(21.4 \%)$ \\
\hline \multicolumn{4}{|l|}{ Tumor size } \\
\hline$<35 \mathrm{~mm}$ & $1134(9.6 \%)$ & $693(8.0 \%)$ & $441(14.2 \%)$ \\
\hline $35-50 \mathrm{~mm}$ & $1039(8.8 \%)$ & $522(6.0 \%)$ & $517(16.7 \%)$ \\
\hline $50-65 \mathrm{~mm}$ & $1241(10.5 \%)$ & $760(8.7 \%)$ & $481(15.5 \%)$ \\
\hline$\geq 65 \mathrm{~mm}$ & $1103(9.3 \%)$ & $639(7.3 \%)$ & $464(14.9 \%)$ \\
\hline Unknown & $7297(61.8 \%)$ & $6096(70.0 \%)$ & $1201(38.7 \%)$ \\
\hline \multicolumn{4}{|l|}{ Presenting features } \\
\hline \multicolumn{4}{|l|}{ HCC risk score } \\
\hline 1st quartile & $2955(25.0 \%)$ & $2232(25.6 \%)$ & $723(23.3 \%)$ \\
\hline 2nd quartile & $2980(25.2 \%)$ & 2079 (23.9\%) & $901(29.0 \%)$ \\
\hline 3rd quartile & $2917(24.7 \%)$ & 2089 (24.0\%) & $828(26.7 \%)$ \\
\hline 4th quartile & $2962(25.1 \%)$ & $2310(26.5 \%)$ & $652(21.0 \%)$ \\
\hline \multicolumn{4}{|l|}{ History of alcoholism } \\
\hline No & $11,390(96.4 \%)$ & 8369 (96.1\%) & $3021(97.3 \%)$ \\
\hline Yes & $424(3.6 \%)$ & $341(3.9 \%)$ & $83(2.7 \%)$ \\
\hline \multicolumn{4}{|l|}{ Tobacco } \\
\hline No & $10,457(88.5 \%)$ & 7690 (88.3\%) & $2767(89.1 \%)$ \\
\hline Yes & $1357(11.5 \%)$ & 1020 (11.7\%) & $337(10.9 \%)$ \\
\hline \multicolumn{4}{|l|}{ History of colorectal polyps } \\
\hline No & $10,459(88.5 \%)$ & 7710 (88.5\%) & $2749(88.6 \%)$ \\
\hline Yes & $1355(11.5 \%)$ & 1000 (11.5\%) & 355 (11.4\%) \\
\hline
\end{tabular}


Table 1 Characteristics of patients with CRC stratified by BO (Continued)

\begin{tabular}{|c|c|c|c|}
\hline Patient characteristics & Overall (N\%) & No BO (N\%) & $\mathrm{BO}(\mathrm{N} \%)$ \\
\hline \multicolumn{4}{|l|}{ Obesity } \\
\hline No & $10,916(92.4 \%)$ & 8045 (92.4\%) & $2871(92.5 \%)$ \\
\hline Yes & $898(7.6 \%)$ & $665(7.6 \%)$ & $233(7.5 \%)$ \\
\hline \multicolumn{4}{|l|}{ Treatment } \\
\hline \multicolumn{4}{|l|}{ Chemotherapy } \\
\hline Nonchemotherapy & $9105(77.1 \%)$ & $6651(76.4 \%)$ & $2454(79.1 \%)$ \\
\hline 5-FU/capecitabine & $1398(11.8 \%)$ & $1047(12.0 \%)$ & $351(11.3 \%)$ \\
\hline FOLFOX/CapeOX & $277(2.3 \%)$ & $213(2.4 \%)$ & $64(2.1 \%)$ \\
\hline FOLFIRI/XELIRI & $242(2.0 \%)$ & $186(2.1 \%)$ & $56(1.8 \%)$ \\
\hline FOLFOX/CapeOX + bevacizumab & $273(2.3 \%)$ & $227(2.6 \%)$ & $46(1.5 \%)$ \\
\hline FOLFIRI/XELIRI + bevacizumab & $42(0.4 \%)$ & $29(0.3 \%)$ & $13(0.4 \%)$ \\
\hline Other & $477(4.0 \%)$ & $357(4.1 \%)$ & $120(3.9 \%)$ \\
\hline \multicolumn{4}{|l|}{ Radiotherapy } \\
\hline No & $9912(83.9 \%)$ & $7252(83.3 \%)$ & $2660(85.7 \%)$ \\
\hline Yes & $1902(16.1 \%)$ & $1458(16.7 \%)$ & $444(14.3 \%)$ \\
\hline \multicolumn{4}{|l|}{ Presenting symptoms } \\
\hline \multicolumn{4}{|l|}{ Abdominal pain } \\
\hline No & $9091(77.0 \%)$ & $6773(77.8 \%)$ & $2318(74.7 \%)$ \\
\hline Yes & $2723(23.0 \%)$ & 1937 (22.2\%) & $786(25.3 \%)$ \\
\hline \multicolumn{4}{|l|}{ Abdominal mass } \\
\hline No & $11,414(96.6 \%)$ & $8431(96.8 \%)$ & $2983(96.1 \%)$ \\
\hline Yes & $400(3.4 \%)$ & $279(3.2 \%)$ & $121(3.9 \%)$ \\
\hline \multicolumn{4}{|l|}{ Abdominal distension } \\
\hline No & $11,624(98.4 \%)$ & $8574(98.4 \%)$ & $3050(98.3 \%)$ \\
\hline Yes & $190(1.6 \%)$ & $136(1.6 \%)$ & $54(1.7 \%)$ \\
\hline \multicolumn{4}{|l|}{ Ascites } \\
\hline No & $11,712(99.1 \%)$ & 8630 (99.1\%) & $3082(99.3 \%)$ \\
\hline Yes & $102(0.9 \%)$ & $80(0.9 \%)$ & $22(0.7 \%)$ \\
\hline \multicolumn{4}{|l|}{ Anemia } \\
\hline No & $10,688(90.5 \%)$ & 7803 (89.6\%) & $2885(92.9 \%)$ \\
\hline Yes & $1126(9.5 \%)$ & $907(10.4 \%)$ & $219(7.1 \%)$ \\
\hline \multicolumn{4}{|l|}{ Nutritional deficiency } \\
\hline No & $11,152(94.4 \%)$ & 8153 (93.6\%) & $2999(96.6 \%)$ \\
\hline Yes & $662(5.6 \%)$ & $557(6.4 \%)$ & $105(3.4 \%)$ \\
\hline \multicolumn{4}{|l|}{ Cachexia } \\
\hline No & $11,742(99.4 \%)$ & 8649 (99.3\%) & $3093(99.6 \%)$ \\
\hline Yes & $72(0.6 \%)$ & $61(0.7 \%)$ & $11(0.4 \%)$ \\
\hline \multicolumn{4}{|l|}{ Change of bowel habits } \\
\hline No & $11,394(96.4 \%)$ & 8395 (96.4\%) & $2999(96.6 \%)$ \\
\hline Yes & $420(3.6 \%)$ & $315(3.6 \%)$ & $105(3.4 \%)$ \\
\hline \multicolumn{4}{|l|}{ Change of character of stool } \\
\hline No & $9536(80.7 \%)$ & 7064 (81.1\%) & $2472(79.6 \%)$ \\
\hline Yes & 2278 (19.3\%) & 1646 (18.9\%) & 632 (20.4\%) \\
\hline
\end{tabular}


Table 1 Characteristics of patients with CRC stratified by BO (Continued)

\begin{tabular}{|c|c|c|c|}
\hline Patient characteristics & Overall (N\%) & No BO (N\%) & $\mathrm{BO}(\mathrm{N} \%)$ \\
\hline \multicolumn{4}{|l|}{ Hemorrhage } \\
\hline No & $9366(79.3 \%)$ & 6859 (78.7\%) & $2507(80.8 \%)$ \\
\hline Yes & $2448(20.7 \%)$ & $1851(21.3 \%)$ & 597 (19.2\%) \\
\hline \multicolumn{4}{|l|}{ Diarrhea } \\
\hline No & 10,899 (92.3\%) & 8022 (92.1\%) & $2877(92.7 \%)$ \\
\hline Yes & $915(7.7 \%)$ & $688(7.9 \%)$ & $227(7.3 \%)$ \\
\hline \multicolumn{4}{|l|}{ Gatism } \\
\hline No & 11,728 (99.3\%) & 8640 (99.2\%) & $3088(99.5 \%)$ \\
\hline Yes & $86(0.7 \%)$ & $70(0.8 \%)$ & $16(0.5 \%)$ \\
\hline \multicolumn{4}{|l|}{ Loss of appetite } \\
\hline No & 11,569 (97.9\%) & 8501 (97.6\%) & $3068(98.8 \%)$ \\
\hline Yes & $245(2.1 \%)$ & 209 (2.4\%) & $36(1.2 \%)$ \\
\hline \multicolumn{4}{|l|}{ Vomiting } \\
\hline No & $11,066(93.7 \%)$ & 8142 (93.5\%) & $2924(94.2 \%)$ \\
\hline Yes & 748 (6.3\%) & $568(6.5 \%)$ & $180(5.8 \%)$ \\
\hline \multicolumn{4}{|l|}{ Weight loss } \\
\hline No & $10,672(90.3 \%)$ & 7838 (90.0\%) & $2834(91.3 \%)$ \\
\hline Yes & 1142 (9.7\%) & 872 (10.0\%) & $270(8.7 \%)$ \\
\hline
\end{tabular}

Abbreviations: CRC colorectal cancer, BO bowel obstruction, HCC the Centers for Medicare and Medicaid Service's Hierarchical Condition Category, 5-FU 5-fluorouracil, FOLFOX 5-FU + oxaliplatin, CapeOX capecitabine + oxaliplatin, FOLFIRI 5-FU + irinotecan, and XELIRI capecitabine + irinotecan. *variable has missing data

and 35-50 mm tumor sizes (HR, 1.266 [95\% CI, 1.1101.444]) had higher cumulative $\mathrm{BO}$ rates. All these factors significantly shortened the time-to-BO, suggesting that they increased the chance of developing $\mathrm{BO}$ in patient survival time. Three presentation features and symptoms remained significant, and abdominal pain (HR, 1.202 [95\% CI, 1.1051.307]) and anemia (HR, 0.802 [95\% CI, 0.696-0.923]) were both positively associated with the onset of BO. In turn, a history of alcoholism seemed to be a protective factor for BO (HR, 0.781 [95\% CI, 0.627-0.973]). In addition, an adjusted HR of 0.591 (95\% CI 0.514-0.679) for BO among patients who received radiotherapy indicated a $40.9 \%$ decrease in the odds of development of BO compared with the nonradiotherapy group. Most types of chemotherapy were effective for $\mathrm{BO}$, and the most effective was 5 -FU + oxaliplatin + bevacizumab [HR, 0.395 (95\% CI, 0.292$0.535)]$ compared to the nonchemotherapy group.

\section{Construction of the prediction tools}

Figure 3 shows the nomogram predicting the 90- and 180-day rates of BO that was constructed based on variables identified as independent factors. We classified the subgroup of variables from low to high by HR and transformed them according to the Cox proportional hazards regression model. The nomogram determines the rate of BO by summing the scores derived from the points scale for each predictor. The calculated score projected to the outcome scale indicates the 90 - and 180-day rates of
BO. The Harrell's C-index of the nomogram was 0.795 (95\% CI, 0.786-0.804). After rectification using a 10-fold cross-validation, the discrimination maintained a C-index of 0.794 .

\section{Discussion}

There is a general consensus about the severity of BO and its intractability. Because of its fatal outcome and poor prognosis $[15,26,27]$, it has become a common palliative indication for surgical consultation [11]. Furthermore, palliative chemotherapy combined with palliative resection has had a better prognosis compared with chemotherapy alone [28]. However, one of the main contradictions for surgery is that patients with $\mathrm{BO}$ often present poor clinical status [12, 29] and high mortality and morbidity in emergency cases [30-33]. A few palliative operations adopted in emergency situations, such as a colostomy [12, 34], which becomes permanent in $40 \%$ of patients [12, 35, 36], can lead to psychological distress for patients [12, 37]. Considering the justification of prophylactic intervention, predicting $\mathrm{BO}$ development is critical in preoperative CRC patients.

Our research focused on the period immediately before tumor resection for all patients. This strategy was intended to identify patients who might develop BO to improve their follow-up or medical intervention. To avoid the effect of surgery, we excluded patients who underwent surgery after CRC diagnosis in the non-BO 
Table 2 Univariate and multivariable analysis of factors associated with BO

\begin{tabular}{|c|c|c|c|c|c|c|}
\hline \multirow[t]{2}{*}{ Patient characteristics } & \multicolumn{3}{|c|}{$\underline{\text { Univariate analysis }}$} & \multicolumn{3}{|c|}{ Multivariate analysis } \\
\hline & $\mathrm{HR}$ & $95 \% \mathrm{Cl}$ & $p$ value & $\mathrm{HR}$ & $95 \% \mathrm{Cl}$ & $p$ value \\
\hline \multicolumn{7}{|l|}{ Gender } \\
\hline Men & 1.087 & $1.013-1.167$ & 0.020 & & & \\
\hline Women & 1.000 & & & & & \\
\hline \multicolumn{7}{|l|}{ Age at diagnosis, years } \\
\hline $66-70$ & 1.818 & $1.642-2.013$ & $<0.001$ & 1.737 & $1.558-1.935$ & $<0.001$ \\
\hline $71-75$ & 1.765 & $1.605-1.942$ & & 1.765 & $1.596-1.953$ & \\
\hline $76-80$ & 1.636 & $1.490-1.796$ & & 1.523 & $1.384-1.677$ & \\
\hline$\geq 81$ & 1.000 & & & 1.000 & & \\
\hline \multicolumn{7}{|l|}{ Race } \\
\hline White & 1.000 & & 0.001 & 1.000 & & 0.004 \\
\hline Black & 0.873 & $0.783-0.972$ & & 0.825 & $0.738-0.922$ & \\
\hline Asian & 1.291 & $1.081-1.542$ & & 1.062 & $0.887-1.271$ & \\
\hline Other & 0.962 & $0.800-1.156$ & & 0.887 & $0.737-1.067$ & \\
\hline \multicolumn{7}{|l|}{ Marital status } \\
\hline Single + separated & 1.067 & $0.945-1.204$ & $<0.001$ & 1.058 & $0.936-1.197$ & $<0.001$ \\
\hline Married & 1.369 & $1.269-1.478$ & & 1.115 & $1.028-1.208$ & \\
\hline Divorced + widowed & 1.000 & & & 1.000 & & \\
\hline Other & 0.565 & $0.468-0.682$ & & 0.622 & $0.514-0.752$ & \\
\hline \multicolumn{7}{|l|}{ Residence location* } \\
\hline Big metro & 1.000 & & $<0.001$ & 1.000 & & 0.050 \\
\hline Metro or urban & 0.863 & $0.797-0.933$ & & 0.906 & $0.837-0.981$ & \\
\hline Less urban or rural & 0.844 & $0.747-0.955$ & & 0.946 & $0.835-1.072$ & \\
\hline \multicolumn{7}{|l|}{ Median household income } \\
\hline 1st quartile & 1.000 & & 0.107 & & & \\
\hline 2nd quartile & 1.066 & $0.961-1.183$ & & & & \\
\hline 3rd quartile & 1.081 & $0.975-1.199$ & & & & \\
\hline 4th quartile & 1.118 & $1.009-1.239$ & & & & \\
\hline Unknown & 0.929 & $0.782-1.104$ & & & & \\
\hline \multicolumn{7}{|l|}{ Level of education } \\
\hline 1st quartile & 1.000 & & 0.571 & & & \\
\hline 2nd quartile & 0.996 & $0.899-1.102$ & & & & \\
\hline 3rd quartile & 0.993 & $0.897-1.100$ & & & & \\
\hline 4th quartile & 0.968 & $0.873-1.072$ & & & & \\
\hline Unknown & 0.872 & $0.735-1.034$ & & & & \\
\hline \multicolumn{7}{|l|}{ Tumor characteristics } \\
\hline \multicolumn{7}{|l|}{ T category } \\
\hline Tis & 1.000 & & $<0.001$ & 1.000 & & $<0.001$ \\
\hline $\mathrm{T} 1$ & 1.532 & $1.233-1.904$ & & 1.434 & $1.144-1.796$ & \\
\hline $\mathrm{T} 2$ & 6.878 & $5.396-8.768$ & & 6.175 & $4.768-7.997$ & \\
\hline T3 & 8.408 & $6.855-10.313$ & & 7.187 & $5.738-9.003$ & \\
\hline $\mathrm{T} 4 \mathrm{a}$ & 15.416 & $11.848-20.059$ & & 9.064 & $6.824-12.039$ & \\
\hline $\mathrm{T} 4 \mathrm{~b}$ & 4.566 & $3.626-5.750$ & & 4.466 & $3.489-5.717$ & \\
\hline Unknown & 1.559 & $1.270-1.913$ & & 1.562 & $1.257-1.941$ & \\
\hline
\end{tabular}


Table 2 Univariate and multivariable analysis of factors associated with BO (Continued)

\begin{tabular}{|c|c|c|c|c|c|c|}
\hline \multirow[t]{2}{*}{ Patient characteristics } & \multicolumn{3}{|c|}{ Univariate analysis } & \multicolumn{3}{|c|}{ Multivariate analysis } \\
\hline & $\mathrm{HR}$ & $95 \% \mathrm{Cl}$ & $p$ value & $\mathrm{HR}$ & $95 \% \mathrm{Cl}$ & $p$ value \\
\hline \multicolumn{7}{|l|}{ M category } \\
\hline Mo & 1.000 & & $<0.001$ & 1.000 & & $<0.001$ \\
\hline M1 & 0.665 & $0.598-0.738$ & & 0.793 & $0.707-0.889$ & \\
\hline Unknown & 0.925 & $0.850-1.007$ & & 1.213 & $1.108-1.328$ & \\
\hline \multicolumn{7}{|l|}{ Primary tumor site } \\
\hline Rectum & 1.000 & & $<0.001$ & 1.000 & & $<0.001$ \\
\hline Left-sided colon & 2.055 & $1.881-2.244$ & & 2.093 & $1.892-2.315$ & \\
\hline Right-sided colon & 1.445 & $1.326-1.574$ & & 1.583 & $1.432-1.750$ & \\
\hline \multicolumn{7}{|l|}{ Histologic type } \\
\hline Adenocarcinoma & 1.000 & & $<0.001$ & 1.000 & & $<0.001$ \\
\hline Mucinous carcinoma & 2.368 & $2.076-2.701$ & & 1.593 & $1.392-1.823$ & \\
\hline Signet-ring cell carcinoma & 2.096 & $1.515-2.899$ & & 1.220 & $0.875-1.701$ & \\
\hline \multicolumn{7}{|l|}{ Histologic grade } \\
\hline Well & 0.771 & $0.669-0.889$ & $<0.001$ & 0.842 & $0.729-0.972$ & $<0.001$ \\
\hline Moderate & 1.000 & & & 1.000 & & \\
\hline Poor & 1.203 & $1.090-1.328$ & & 1.131 & $1.022-1.251$ & \\
\hline Undifferentiated & 0.954 & $0.652-1.395$ & & 0.992 & $0.676-1.456$ & \\
\hline Unknown & 0.383 & $0.350-0.419$ & & 0.548 & $0.498-0.604$ & \\
\hline \multicolumn{7}{|l|}{ Tumor size, mm } \\
\hline$<35$ & 1.000 & & $<0.001$ & 1.000 & & $<0.001$ \\
\hline $35-50$ & 1.734 & $1.526-1.970$ & & 1.266 & $1.110-1.444$ & \\
\hline $50-65$ & 1.269 & $1.114-1.446$ & & 1.133 & $0.991-1.295$ & \\
\hline$\geq 65$ & 1.543 & $1.353-1.759$ & & 1.253 & $1.093-1.436$ & \\
\hline Unknown & 0.419 & $0.376-0.467$ & & 0.616 & $0.549-0.690$ & \\
\hline \multicolumn{7}{|l|}{ Presenting features } \\
\hline \multicolumn{7}{|l|}{ HCC risk score } \\
\hline 1st quartile & 1.000 & & $<0.001$ & & & \\
\hline 2nd quartile & 1.187 & $1.076-1.309$ & & & & \\
\hline 3rd quartile & 1.126 & $1.019-1.245$ & & & & \\
\hline 4th quartile & 0.913 & $0.821-1.015$ & & & & \\
\hline \multicolumn{7}{|l|}{ History of alcoholism } \\
\hline No & 1.000 & & 0.011 & 1.000 & & 0.027 \\
\hline Yes & 0.753 & $0.606-0.937$ & & 0.781 & $0.627-0.973$ & \\
\hline \multicolumn{7}{|l|}{ Tobacco } \\
\hline No & 1.000 & & 0.385 & & & \\
\hline Yes & 0.951 & $0.849-1.065$ & & & & \\
\hline \multicolumn{7}{|l|}{ History of colorectal polyps } \\
\hline No & 1.000 & & 0.004 & & & \\
\hline Yes & 0.850 & $0.760-0.949$ & & & & \\
\hline \multicolumn{7}{|l|}{ Obesity } \\
\hline No & 1.000 & & 0.845 & & & \\
\hline Yes & 0.987 & $0.863-1.128$ & & & & \\
\hline
\end{tabular}


Table 2 Univariate and multivariable analysis of factors associated with BO (Continued)

\begin{tabular}{|c|c|c|c|c|c|c|}
\hline \multirow[t]{2}{*}{ Patient characteristics } & \multicolumn{3}{|c|}{ Univariate analysis } & \multicolumn{3}{|c|}{ Multivariate analysis } \\
\hline & $\mathrm{HR}$ & $95 \% \mathrm{Cl}$ & $p$ value & $\mathrm{HR}$ & $95 \% \mathrm{Cl}$ & $p$ value \\
\hline \multicolumn{7}{|l|}{ Treatment } \\
\hline \multicolumn{7}{|l|}{ Chemotherapy } \\
\hline Nonchemotherapy & 1.000 & & $<0.001$ & 1.000 & & $<0.001$ \\
\hline 5-FU/capecitabine & 0.758 & $0.677-0.847$ & & 0.752 & $0.655-0.864$ & \\
\hline FOLFOX/CapeOX & 0.719 & $0.561-0.922$ & & 0.595 & $0.459-0.770$ & \\
\hline FOLFIRI/XELIRI & 0.729 & $0.559-0.950$ & & 0.629 & $0.480-0.825$ & \\
\hline FOLFOX/CapeOX + bevacizumab & 0.482 & $0.360-0.645$ & & 0.395 & $0.292-0.535$ & \\
\hline FOLFIRI/XELIRI + bevacizumab & 0.921 & $0.534-1.589$ & & 0.980 & $0.564-1.705$ & \\
\hline Other & 0.798 & $0.664-0.958$ & & 0.715 & $0.590-0.867$ & \\
\hline \multicolumn{7}{|l|}{ Radiotherapy } \\
\hline No & 1.000 & & $<0.001$ & 1.000 & & $<0.001$ \\
\hline Yes & 0.705 & $0.637-0.780$ & & 0.591 & $0.514-0.679$ & \\
\hline \multicolumn{7}{|l|}{ Presenting symptoms } \\
\hline \multicolumn{7}{|l|}{ Abdominal pain } \\
\hline No & 1.000 & & $<0.001$ & & & $<0.001$ \\
\hline Yes & 1.179 & $1.087-1.278$ & & 1.202 & $1.105-1.307$ & \\
\hline \multicolumn{7}{|l|}{ Abdominal mass } \\
\hline No & 1.000 & & 0.025 & 1.000 & & 0.056 \\
\hline Yes & 1.230 & $1.026-1.476$ & & 1.199 & $0.996-1.445$ & \\
\hline \multicolumn{7}{|l|}{ Abdominal distension } \\
\hline No & 1.000 & & 0.381 & & & \\
\hline Yes & 1.128 & $0.862-1.476$ & & & & \\
\hline \multicolumn{7}{|l|}{ Ascites } \\
\hline No & 1.000 & & 0.497 & & & \\
\hline Yes & 0.865 & $0.569-1.315$ & & & & \\
\hline \multicolumn{7}{|l|}{ Anemia } \\
\hline No & 1.000 & & $<0.001$ & 1.000 & & 0.002 \\
\hline Yes & 0.736 & $0.642-0.845$ & & 0.802 & $0.696-0.923$ & \\
\hline \multicolumn{7}{|l|}{ Nutritional deficiency } \\
\hline No & 1.000 & & $<0.001$ & 1.000 & & 0.067 \\
\hline Yes & 0.624 & $0.513-0.758$ & & 0.830 & $0.680-1.013$ & \\
\hline \multicolumn{7}{|l|}{ Cachexia } \\
\hline No & 1.000 & & 0.166 & & & \\
\hline Yes & 0.658 & $0.364-1.189$ & & & & \\
\hline \multicolumn{7}{|l|}{ Change of bowel habit } \\
\hline No & 1.000 & & 0.023 & & & \\
\hline Yes & 0.798 & $0.657-0.969$ & & & & \\
\hline \multicolumn{7}{|l|}{ Change of character of stool } \\
\hline No & 1.000 & & 0.348 & & & \\
\hline Yes & 1.043 & $0.955-1.138$ & & & & \\
\hline \multicolumn{7}{|l|}{ Hemorrhage } \\
\hline No & 1.000 & & $<0.001$ & & & \\
\hline Yes & 0.841 & $0.769-0.920$ & & & & \\
\hline
\end{tabular}


Table 2 Univariate and multivariable analysis of factors associated with BO (Continued)

\begin{tabular}{|c|c|c|c|c|c|c|}
\hline \multirow[t]{2}{*}{ Patient characteristics } & \multicolumn{3}{|c|}{ Univariate analysis } & \multicolumn{3}{|c|}{ Multivariate analysis } \\
\hline & $\overline{\mathrm{HR}}$ & $95 \% \mathrm{Cl}$ & $p$ value & $\mathrm{HR}$ & $95 \% \mathrm{Cl}$ & $p$ value \\
\hline \multicolumn{7}{|l|}{ Diarrhea } \\
\hline No & 1.000 & & 0.458 & & & \\
\hline Yes & 0.950 & $0.830-1.088$ & & & & \\
\hline \multicolumn{7}{|l|}{ Gatism } \\
\hline No & 1.000 & & 0.183 & & & \\
\hline Yes & 0.716 & $0.438-1.170$ & & & & \\
\hline \multicolumn{7}{|l|}{ Loss of appetite } \\
\hline No & 1.000 & & 0.002 & 1.000 & & 0.077 \\
\hline Yes & 0.598 & $0.431-0.831$ & & 0.742 & $0.533-1.033$ & \\
\hline \multicolumn{7}{|l|}{ Vomiting } \\
\hline No & 1.000 & & 0.537 & & & \\
\hline Yes & 0.954 & $0.820-1.109$ & & & & \\
\hline \multicolumn{7}{|l|}{ Weight loss } \\
\hline No & 1.000 & & 0.579 & & & \\
\hline Yes & 0.965 & $0.852-1.094$ & & & & \\
\hline
\end{tabular}

Abbreviations: $B O$ bowel obstruction, $\mathrm{HCC}$ the Centers for Medicare and Medicaid Service's Hierarchical Condition Category, $H R$ hazard ratio, $C l$ confidence intervals, 5 - $F U$ 5-fluorouracil, FOLFOX 5-FU + oxaliplatin, CapeOX capecitabine + oxaliplatin, FOLFIRI 5-FU + irinotecan, and XELIRI capecitabine + irinotecan. *variable has missing data

A

1.0 Age at diagnosis, years

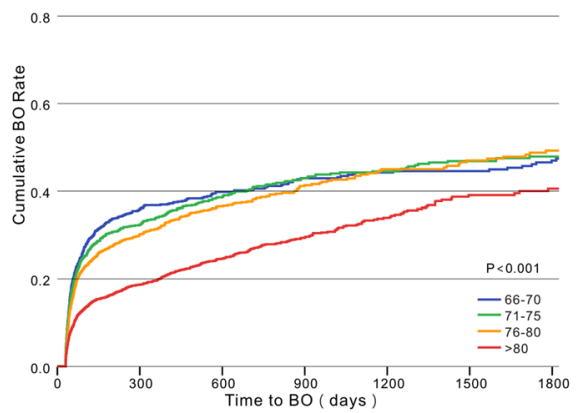

C M category

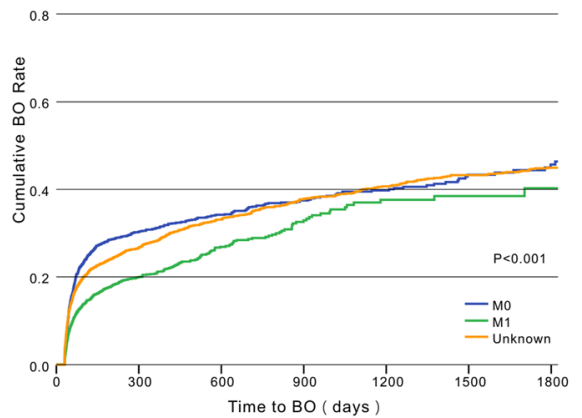

B 1.0 T category

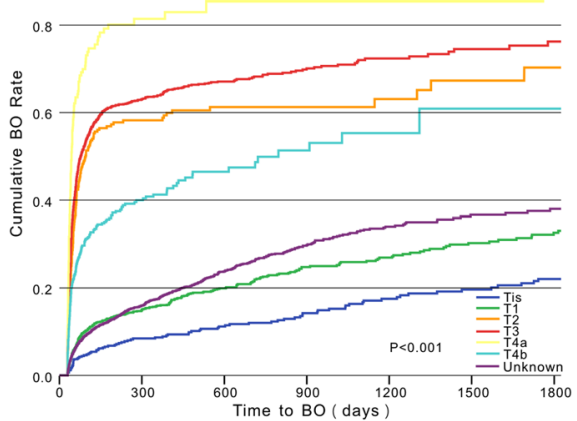

D Primary tumor site

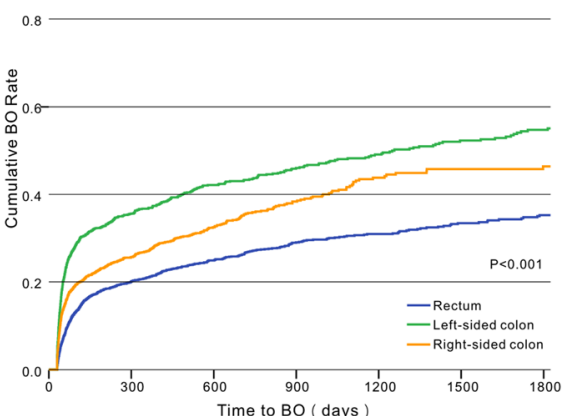

Fig. 1 a Kaplan-Meier analysis of time-to-BO stratified by age among the patients with CRC. $\mathbf{b}$ Kaplan-Meier analysis of time-to-BO stratified by T category among the patients with CRC. $\mathbf{c}$ Kaplan-Meier analysis of time-to-BO stratified by M category among the patients with CRC. $\mathbf{d}$ Kaplan-Meier analysis of time-to-BO stratified by primary tumor site among the patients with CRC 

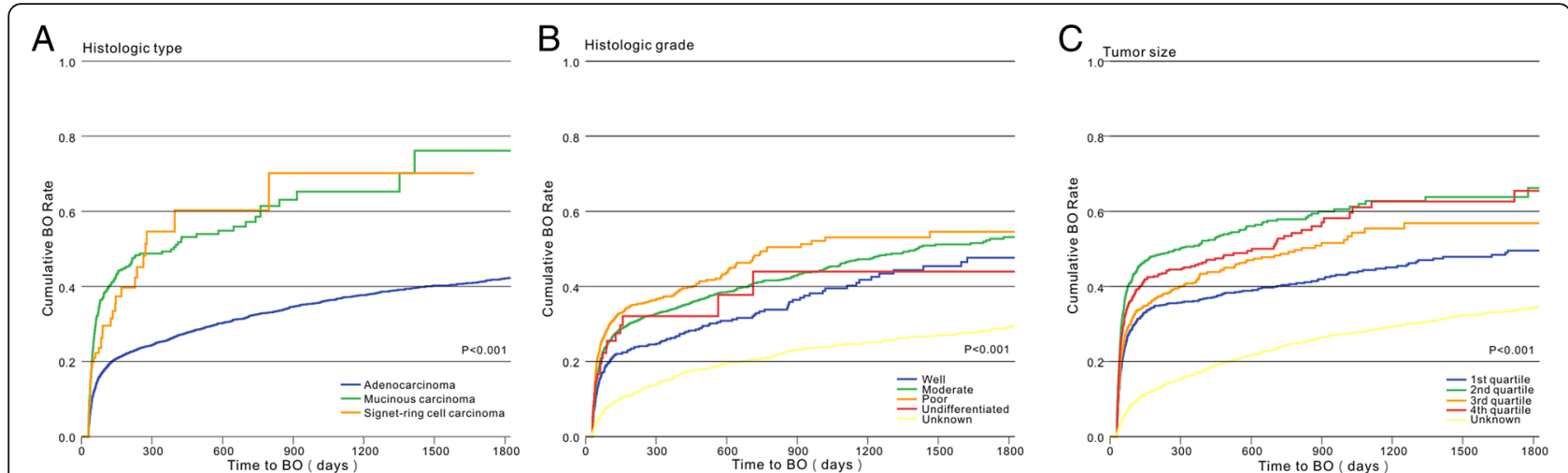

Fig. 2 a Kaplan-Meier analysis of time-to-BO stratified by histologic type among the patients with CRC. b Kaplan-Meier analysis of time-to-BO stratified by histologic grade among the patients with CRC. c Kaplan-Meier analysis of time-to-BO stratified by tumor size among the patients with CRC

group and patients who received surgery after CRC diagnosis and before recorded $\mathrm{BO}$ in the $\mathrm{BO}$ group. We chose patients who did not present with $\mathrm{BO}$ at the inception of the study. Fourteen factors derived from four classifications, including patient characteristics, tumor characteristics, presentation features and symptoms, and treatment, were associated with $\mathrm{BO}$. All these factors were used to construct a nomogram and provide a score to predict the individual probability of developing $\mathrm{BO}$. In contrast, the factors described in other studies,

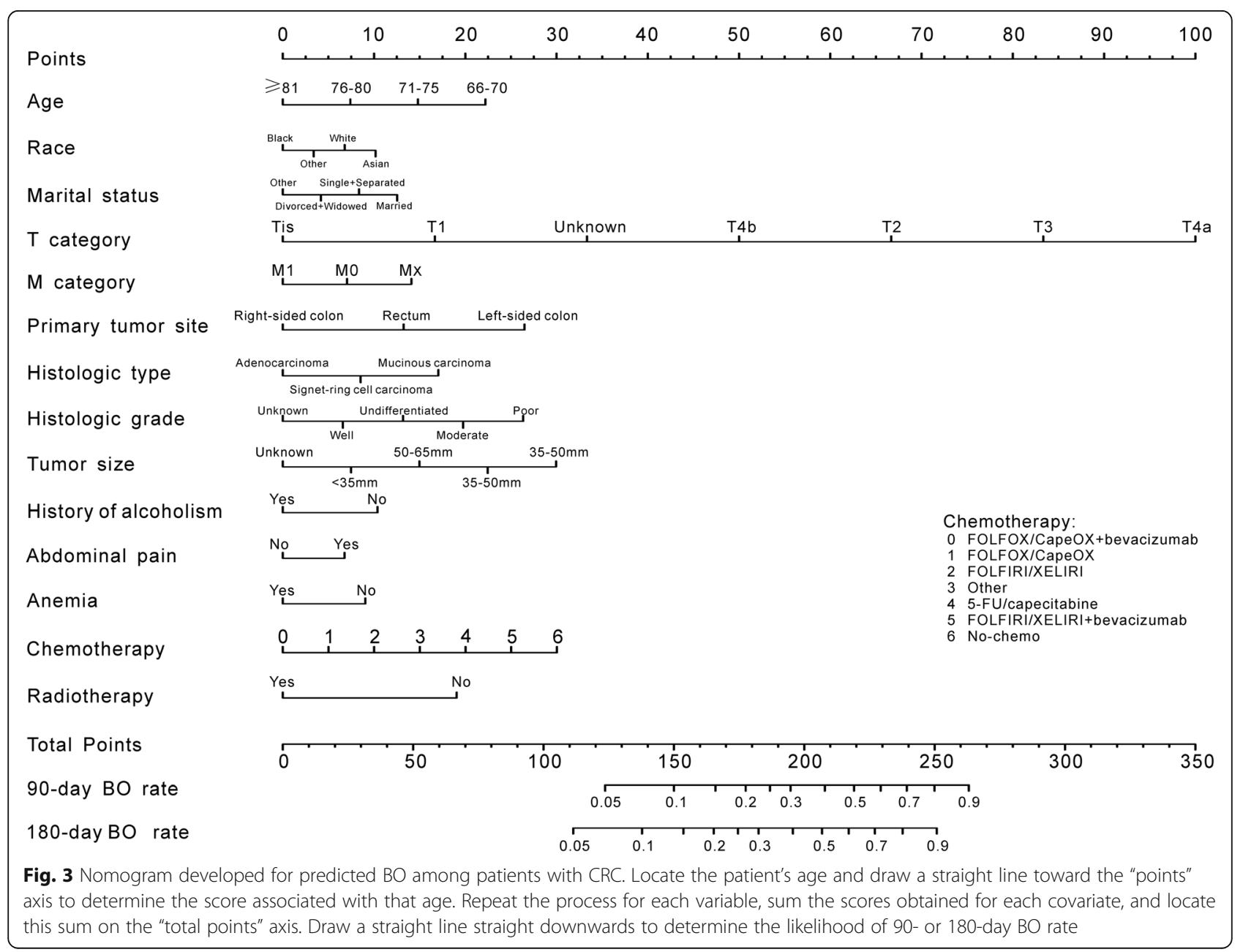


including female sex, high comorbidity score, living in urban areas, and low income [19], played no role in our study.

Younger age was associated with an increased probability of BO, which may be explained by the shorter survival time among the older age groups, considering that the risk of $\mathrm{BO}$ was inversely correlated with death. Other studies reported similar results [19, 21]. Winner et al. [21] indicated that death is a competing outcome associated with BO. The time-to-BO model we used censored death; therefore, the shorter survival time could explain the decreased risk of $\mathrm{BO}$ among older patients as demonstrated in the epidemiologic study conducted by Lau et al. [38]. The analysis of other patient characteristics indicated that married subjects and Asians were more likely to develop BO.

Previous studies presented inconsistent results regarding the relationship between $\mathrm{BO}$ development and different primary tumor sites, including the right colon [18], descending colon [39], and sigmoid colon [40, 41]. Our results indicated that the left-sided colon (HR 2.093 [95\% CI, 1.892-2.315]) was more susceptible to BO compared with the right-sided colon (HR 1.583 [95\% CI, 1.432-1.750]) and the rectum (HR 1); similar results were obtained by Rebeneck et al. [19, 32].

In the $\mathrm{T}$ category, the higher (T4a) group developed BO more frequently (HR, 9.064 [95\% CI, 6.824-12.039]). A possible explanation is that the higher the $\mathrm{T}$ category, the deeper the infiltrate. The increased thickness of the bowel wall prevents the movement of the bowel content. Of note, the T4b group had an even lower risk of BO than the T2 group, which cannot be supported by the currently proposed mechanism. Therefore, we hypothesize that T4b tumors tend to be exophytic and spread beyond the gut epithelium. A similar phenomenon was that patients with tumor sizes of $35-50 \mathrm{~mm}$ had the highest risk of $\mathrm{BO}$. This result disagrees with our previous assumption that the larger the tumor, the higher the likelihood of developing BO.

Our results indicated that M1 (HR 0.793 [95\% CI, 0.707-0.889]) had a lower risk than M0 (HR 1). A previous study suggested that the risk of developing $\mathrm{BO}$ did not appear to be higher for stage IV disease than for earlier stages [42]. We propose that the management of patients with $\mathrm{BO}$ and metastatic disease is different from that of patients with localized disease. Intensive chemotherapy regimens may decrease the incidence of BO. Another hypothesis is that these results are due to a shorter survival time.

We also found that the histologic type and grade played a role in the onset of $\mathrm{BO}$. Mucinous carcinoma (HR 1.593 [95\% CI, 1.392-1.823]) and signet-ring cell carcinoma (HR 1.220 [95\% CI, 0.875-1.701]) increased the risk of development of $\mathrm{BO}$ compared with adenocarcinoma. Poor differentiation can also increase the risk of BO. Significant differences in epidemiologic, clinical, pathological, and molecular phenotypes were found between adenocarcinoma and non-adenocarcinoma, as well as between lower-differentiation and higher-differentiation grades. We propose that the effect of these two factors was correlated with the molecular entity and its subsequent influence. Mucinous and poorly differentiated CRC tumors tend to be infiltrative and more aggressive and have a poorer prognosis [43]. These characteristics increase both tumor resistance to medical treatment and the risk of $\mathrm{BO}$.

The efficacy of chemotherapy and radiotherapy was also evaluated in our study. The risk of $\mathrm{BO}$ in the chemotherapy groups was lower than that in the nonchemotherapy group. We propose that systemic chemotherapy reduces tumor burden.

Symptoms and features that were not considered relevant in previous studies were found to be associated with $\mathrm{BO}$ in our study, including abdominal pain (HR 1.202 [95\% CI, 1.105-1.307]), which is often the first symptom presented at diagnosis. In addition, anemia and a history of alcoholism appear to be protective factors for BO. This result was not expected because alcohol consumption is considered a risk factor for left-sided colon cancer $[44,45]$ and, as indicated earlier in this study, left-sided-colon tumor location increased the risk of BO.

In clinical practice, we are more concerned about improving screening and providing more aggressive treatment to patients at a high risk for $\mathrm{BO}$, which requires highly accurate diagnostic methods. The nomogram constructed to predict BO had a C-index of 0.795 [95\% $\mathrm{CI}, 0.786-0.804]$, indicating a moderate prediction capability in the derivation set. A 10-fold cross-validation was adopted to reduce overfitting and assess the stability of predictive ability of the model. The verification result, a C-index of 0.794 , demonstrates that the results were reproducible and suggests the potential clinical application of this index.

This study has several limitations, including its retrospective design and the possible misclassification of patients because of coding errors. The $\mathrm{T}$ and $\mathrm{M}$ categories in the nonsurgical patients were based on imaging examinations or remained unknown. Thus, misclassifications might have been corrected by pathological reports for the patients who underwent surgery after $\mathrm{BO}$. The different classification sources were confounding factors. $\mathrm{N}$ category was not included in our study because most of our population did not undergo cancer-related surgery and the exact nodal stage remained unknown. Moreover, for generalized use of the nomogram by other institutions or other regions, it is important to minimize the effect of differences. So, it is necessary for a 
prospective evaluation of the presented nomogram and its applicability in clinical setting.

\section{Conclusions}

We found that 14 factors were associated with $\mathrm{BO}$, and these factors were used to build a nomogram. To the best of our knowledge, this study is the first to make a large-scale, population-based assessment of $\mathrm{BO}$ in preoperative patients with CRC. Moreover, this statistical model is the first to predict the development of $\mathrm{BO}$ in preoperative CRC patients. The present study may advance the ability of surgeons to make decisions on the best intervention for patients at risk for $\mathrm{BO}$.

\section{Additional file}

Additional file 1: Table S1. Translation of symptoms involved in study into ICD-9-CM codes. (DOCX $13 \mathrm{~kb}$ )

Additional file 2: Table S2. The health care financing administration common procedure coding system or national drug code for treatment. (DOCX $14 \mathrm{~kb}$ )

\section{Abbreviations}

AJCC: American Joint Committee on Cancer; BO: Bowel obstruction; Cl: Confidence intervals; CRC: Colorectal cancer; FOLFIRI/XELIRI: 5-FU/ capecitabine plus irinotecan; FOLFOX/CapeOX: 5-FU/capecitabine plus oxaliplatin; HCC: Hierarchical condition category; HMO: Health maintenance organization; HR: Hazard ratio; ICD-9: International Classification of Diseases, Ninth Revision; SEER: National Cancer Institute Surveillance, Epidemiology, and End Results; SEER-M database: Surveillance, Epidemiology, and End Results and Medicare claims-linked databases

\section{Acknowledgements}

Not applicable

\section{Funding}

This work was funded by National Key R\&D Program of China (MOST2016YFC1303200, MOST-2016YFC1303202), Clinical Capability Construction Project for Liaoning Provincial Hospitals (LNCCC-A01-2014), and Key Laboratory Programme of Education Department of Liaoning Province (LZ2015076).

\section{Availability of data and materials}

This retrospective study used data from the National Cancer Institute Surveillance, Epidemiology, and End Results (SEER) registry linked with Medicare claims data [16]. The SEER database is a population-based cancer registry covering approximately $28 \%$ of the population across the United States [17]. Medicare claims files from the Centers for Medicare and Medicaid is the primary health insurer for approximately $97 \%$ of the population of the Unites States aged $\geq 65$ years [16].

\section{Authors' contributions}

$X L$ helped in the conception, design, and writing of the study. $H Y, X C$, and YW helped in drafting the article; PG helped in the data curation and analysis; YS helped in the formal analysis; JS helped in the methodology and interpretation of data; ZW helped in the conception, design, funding acquisition and project administration. All authors read and approved the final manuscript.

\section{Ethics approval and consent to participate}

The authors obtained the permission to analyzed data from SEER-Medicare program with the reference number D6-MEDIC-821, and masked the information could be linked to individual patients. The China Medical University's institutional review board approved this study and the reference number was [2012]96.

\section{Consent for publication}

Not applicable.

\section{Competing interests}

The authors declare that they have no competing interests.

\section{Publisher's Note}

Springer Nature remains neutral with regard to jurisdictional claims in published maps and institutional affiliations.

Received: 1 October 2018 Accepted: 3 January 2019

Published online: 18 January 2019

\section{References}

1. Siegel RL, Miller KD, Fedewa SA, Ahnen DJ, Meester RGS, et al. Colorectal cancer statistics, 2017. CA Cancer J Clin. 2017;67:177-93.

2. Ahuja N, Chang D, Gearhart SL. Disparities in colon cancer presentation and in-hospital mortality in Maryland: a ten-year review. Ann Surg Oncol. 2007; 14:411-6.

3. Umpleby HC, Bristol JB, Rainey JB, Williamson RC. Survival of 727 patients with single carcinomas of the large bowel. Dis Colon Rectum. 1984;27:803-10.

4. Alese OB, Kim S, Chen Z, Owonikoko TK, El-Rayes BF. Management patterns and predictors of mortality among US patients with cancer hospitalized for malignant bowel obstruction. Cancer. 2015;121:1772-8.

5. Baines M, Oliver DJ, Carter RL. Medical management of intestinal obstruction in patients with advanced malignant disease. A clinical and pathological study. Lancet. 1985;2:990-3.

6. Mooney SJ, Winner M, Hershman DL, Wright JD, Feingold DL, et al. Bowel obstruction in elderly ovarian cancer patients: a population-based study. Gynecol Oncol. 2013;129:107-12.

7. Ripamonti Cl, Easson AM, Gerdes H. Management of malignant bowel obstruction. Eur J Cancer. 2008;44:1105-15.

8. Laval G, Girardier J, Lassauniere JM, Leduc B, Haond C, et al. The use of steroids in the management of inoperable intestinal obstruction in terminal cancer patients: do they remove the obstruction? Palliat Med. 2000;14:3-10.

9. Feuer DJ, Broadley KE, Shepherd JH, Barton DP. Systematic review of surgery in malignant bowel obstruction in advanced gynecological and gastrointestinal cancer. The Systematic Review Steering Committee. Gynecol Oncol. 1999;75:313-22.

10. Borneman T, Chu DZ, Wagman L, Ferrell B, Juarez G, et al. Concerns of family caregivers of patients with cancer facing palliative surgery for advanced malignancies. Oncol Nurs Forum. 2003;30:997-1005.

11. Lilley EJ, Scott JW, Goldberg JE, Cauley CE, Temel JS, et al. Survival, healthcare utilization, and end-of-life care among older adults with malignancy-associated bowel obstruction: comparative study of surgery, venting gastrostomy, or medical management. Ann Surg. 2018;267(4):692-99.

12. Jimenez-Perez J, Casellas J, Garcia-Cano J, Vandervoort J, Garcia-Escribano $\mathrm{OR}$, et al. Colonic stenting as a bridge to surgery in malignant large-bowel obstruction: a report from two large multinational registries. Am J Gastroenterol. 2011;106:2174-180

13. Legendre H, Vanhuyse F, Caroli-BosC FX, Pector JC. Survival and quality of life after palliative surgery for neoplastic gastrointestinal obstruction. Eur J Surg Oncol. 2001;27:364-7.

14. Makela J, Kiviniemi H, Laitinen S, Kairaluoma MI. Surgical management of intestinal obstruction after treatment for cancer. Case reports. Eur J Surg. 1991;157:73-7.

15. Chakraborty A, Selby D, Gardiner K, Myers J, Moravan V, et al. Malignant bowel obstruction: natural history of a heterogeneous patient population followed prospectively over two years. J Pain Symptom Manag. 2011;41:412-20.

16. Potosky AL, Riley GF, Lubitz JD, Mentnech RM, Kessler LG. Potential for cancer related health services research using a linked Medicare-tumor registry database. Med Care. 1993;31:732-48.

17. National Cancer Institute. Surveillance, Epidemiology and End Results (SEER). 2015. http://seer.cancer.gov/about/overview.html. Accessed 27 Apr 2017.

18. Rabeneck L, Paszat LF, Li C. Risk factors for obstruction, perforation, or emergency admission at presentation in patients with colorectal cancer: a population-based study. Am J Gastroenterol. 2006;101:1098-103.

19. Dominitz JA, Samsa GP, Landsman P, Provenzale D. Race, treatment, and survival among colorectal carcinoma patients in an equal-access medical system. Cancer 1998;82:2312-20.

20. Winner M, Mooney SJ, Hershman DL, Feingold DL, Allendorf JD, et al. Incidence and predictors of bowel obstruction in elderly patients with stage IV colon cancer: a population-based cohort study. JAMA Surg. 2013;148: $715-22$. 
21. Kim DH, Kim B, Choi JH, Park SJ, Hong SP, Cheon JH, et al. Tumor characteristics associated with malignant large bowel obstruction in stage IV colorectal cancer patients undergoing chemotherapy. Int J Colorectal Dis. 2016:31(11):1767-74. https://doi.org/10.1007/s00384-016-2638-y.

22. Amin MB, Greene FL, Edge SB, et al. AJCC Cancer Staging Manual. 8th ed. New York: Springer; 2016.AQ10

23. Gao $P$, Song $Y X$, Sun JX, Chen XW, Xu YY, et al. Which is the best postoperative chemotherapy regimen in patients with rectal cancer after neoadjuvant therapy? BMC Cancer. 2014;14:888.

24. Ash AS, Ellis RP, Pope GC, Ayanian JZ, Bates DW, et al. Using diagnoses to describe populations and predict costs. Health Care Financ Rev. 2000;21:7-28

25. Delen D, Walker G, Kadam A. Predicting breast cancer survivability: a comparison of three data mining methods. Artif Intell Med. 2005;34:113-27.

26. Selby D, Wright F, Stilos K, Daines P, Moravan V, et al. Room for improvement? A quality-of-life assessment in patients with malignant bowe obstruction. Palliat Med. 2010;24:38-45.

27. Mirensky TL, Schuster KM, Ali UA, Reddy V, Schwartz PE, et al. Outcomes of small bowel obstruction in patients with previous gynecologic malignancies. Am J Surg. 2012;203:472-9.

28. Park JH, Kim TY, Lee KH, Han SW, Oh DY, et al. The beneficial effect of palliative resection in metastatic colorectal cancer. Br J Cancer. 2013;108; 1425-31.

29. Soto S, Lopez-Roses L, Gonzalez-Ramirez A, Lancho A, Santos A, et al. Endoscopic treatment of acute colorectal obstruction with self-expandable metallic stents: experience in a community hospital. Surg Endosc. 2006;20: 1072-6.

30. Paulson EC, Mahmoud NN, Wirtalla C, Armstrong K. Acuity and survival in colon cancer surgery. Dis Colon Rectum. 2010;53:385-92.

31. Diggs JC, Xu F, Diaz M, Cooper GS, Koroukian SM. Failure to screen: predictors and burden of emergency colorectal cancer resection. Am J Manag Care. 2007;13:157-64.

32. Phillips RK, Hittinger R, Fry JS, Fielding LP. Malignant large bowel obstruction. Br J Surg. 1985;72:296-302.

33. Ohman U. Prognosis in patients with obstructing colorectal carcinoma. Am J Surg. 1982;143:742-7.

34. Scott NA, Jeacock J, Kingston RD. Risk factors in patients presenting as an emergency with colorectal cancer. Br J Surg. 1995;82:321-3.

35. Watt AM, Faragher IG, Griffin TT, Rieger NA, Maddern GJ. Self-expanding metallic stents for relieving malignant colorectal obstruction: a systematic review. Ann Surg. 2007;246:24-30,

36. Kim JS, Hur H, Min BS, Sohn SK, Cho CH, et al. Oncologic outcomes of self-expanding metallic stent insertion as a bridge to surgery in the management of left-sided colon cancer obstruction: comparison with nonobstructing elective surgery. World J Surg. 2009;33:1281-6.

37. Karadag A, Mentes BB, Uner A, Irkorucu O, Ayaz S, et al. Impact of stomatherapy on quality of life in patients with permanent colostomies or ileostomies. Int J Color Dis. 2003;18:234-8.

38. Lau B, Cole SR, Gange SJ. Competing risk regression models for epidemiologic data. Am J Epidemiol. 2009;170:244-56.

39. Wolmark N, Wieand HS, Rockette HE, Fisher B, Glass A, et al. The prognostic significance of tumor location and bowel obstruction in Dukes B and C colorectal cancer. Findings from the NSABP clinical trials. Ann Surg. 1983; 198:743-52.

40. Sarela Al, Guthrie JA, Seymour MT, Ride E, Guillou PJ, et al. Non-operative management of the primary tumour in patients with incurable stage IV colorectal cancer. Br J Surg. 2001;88:1352-6.

41. Scoggins CR, Meszoely IM, Blanke CD, Beauchamp RD, Leach SD. Nonoperative management of primary colorectal cancer in patients with stage IV disease. Ann Surg Oncol. 1999;6:651-7.

42. Stamatakis J, Thompson M, Chave H, Kinsman R. National audit of bowel obstruction due to colorectal cancer: April 1998-March 1999. London: The Association of Coloproctology of Great Britain and Ireland; 2002.

43. Park YJ, Park KJ, Park JG, Lee KU, Choe KJ, et al. Prognostic factors in 2230 Korean colorectal cancer patients: analysis of consecutively operated cases. World J Surg. 1999;23:721-6.

44. Toyomura K, Yamaguchi K, Kawamoto H, Tabata S, Shimizu E, et al. Relation of cigarette smoking and alcohol use to colorectal adenomas by subsite: the self-defense forces health study. Cancer Sci. 2004;95:72-6.

45. Nawa T, Kato J, Kawamoto H, Okada H, Yamamoto H, et al. Differences between right- and left-sided colon cancer in patient characteristics, cancer morphology and histology. J Gastroenterol Hepatol. 2008;23:418-23.

Ready to submit your research? Choose BMC and benefit from:

- fast, convenient online submission

- thorough peer review by experienced researchers in your field

- rapid publication on acceptance

- support for research data, including large and complex data types

- gold Open Access which fosters wider collaboration and increased citations

- maximum visibility for your research: over $100 \mathrm{M}$ website views per year

At BMC, research is always in progress.

Learn more biomedcentral.com/submissions 